Décadrages Décadrages

cınéma, à travers champs Cinéma, à travers champs

15 | 2009

Raoul Ruiz

\title{
Quelques jours avant la nuit... (2008) de Simon Edelstein ou les dérives de la critique romande
}

\section{Marthe Porret}

\section{OpenEdition}

Journals

Édition électronique

URL : http://journals.openedition.org/decadrages/128

DOI : $10.4000 /$ decadrages. 128

ISSN : 2297-5977

Éditeur

Association Décadrages

Édition imprimée

Date de publication : 1 octobre 2009

Pagination : 110-117

ISBN : 978-2-9700668-0-4

ISSN : 2235-7823

Référence électronique

Marthe Porret, « Quelques jours avant la nuit... (2008) de Simon Edelstein ou les dérives de la critique romande », Décadrages [En ligne], 15 | 2009, mis en ligne le 26 novembre 2012, consulté le 01 mai 2019. URL : http://journals.openedition.org/decadrages/128; DOI : 10.4000/decadrages.128

(B) Décadrages 


\section{Quelques jours avant la nuit... (2008) de Simon Edelstein ou les dérives de la critique romande}

1 Norbert Creutz, "Petits mystères d'un soir", Le Temps, 3.11.2008.

2 Simon Edelstein est l'auteur d'une dizaine de livres de photographies pour les Editions Rencontre et La Guilde du livre à Lausanne.

3 Voir par exemple l'Histoire du cinéma suisse 1966-2000 d'Hervé Dumont et Maria Tortajada (dir.), Cinémathèque suisse/Editions Gilles Attinger, Lausanne/Hauterive, 2007, t. I, p. 154.

4 Les Vilaines manières (1973), Un homme en fuite (1979), L'Ogre (1986), Passage au crépuscule (2000).

5 Norbert Creutz, op. cit. par Marthe Porret

"Quelques jours avant la nuit de Simon Edelstein opte pour l'arrièregarde. Même si on ne sait rien du parcours de Simon Edelstein, son nouveau film a déjà de quoi laisser songeur. Pour preuve, ni les festivals, ni les distributeurs ne se sont bousculés au portillon...

[...] Le point de vue est flottant, la mise en scène superficielle, l'ironie absente. Bref, on se situe strictement au rayon cinéma de papa'.» 1

C'est ainsi que Norbert Creutz ouvre et conclut les feux de sa critique. La rhétorique est adroite: partant du principe que les lecteurs n'ont jamais entendu parler de Simon Edelstein, le journaliste ne se donne pas la peine de nous informer plus avant. Pourtant le cinéaste genevois Simon Edelstein n'est pas un inconnu, ni un nouveau venu. Photographe ${ }^{2}$, entré à la TSR comme caméraman en 1966, il va s'y imposer comme réalisateur en signant plus de cent émissions (Spécial Cinéma, Passe-moi les jumelles, Viva, Temps présent,...). Mais c'est aussi en tant que chef opérateur qu'il va travailler, notamment avec Michel Soutter (La Pomme, 1969; James ou pas, 1970 ; Les Arpenteurs, 1972; L’Escapade, 1974) ${ }^{3}$. Quelques clicks sur le net suffisent pour voir qu'il est l'auteur, dès 1973, de plusieurs longs métrages de fiction 4 .

Ce qui frappe d'emblée ici, c'est l'arbitraire de l'«analyse» critique proprement dite. Premier constat: le film est mauvais car il n'a pas trouvé preneur... Cinéphile chevronné, Creutz sait bien que nombre de «chefd'œuvres » de l'histoire du cinéma n'ont jamais connu de distribution commerciale. Deuxième constat: décrétant que le film relève d'un genre précis, celui "du thriller psychologique dans une grande demeure», le journaliste ajoute: "Intéressant dans l'optique d'un budget serré, [ce film de genre] doit encore devenir captivant pour le spectateur. On est hélas loin du compte dans cette histoire un peu languissante d'une pianiste $[\ldots] » 5$. La formulation dévoile à quel point la réception des films chez le journaliste dépend d'une grille de lecture normative aussi bien 
du point de vue de la narration (le récit ne doit pas être ennuyeux) que des pratiques de production (le huis clos s'impose quand le budget est modeste).

\section{Historique de production}

Or, lorsqu'on se penche sur l'histoire de ce film, on se rend compte que l'idée de ce huis clos a été posée des années avant que le montage de la production ne soit bouclé. Le budget n'a donc pas déterminé le genre dont relève ce film. Ainsi, il vaut la peine de revenir sur la genèse de Quelques jours avant la nuit... pour mettre en évidence les conditions de production qui ont déterminé, comme nous allons le voir, la carrière de ce film.

De la rencontre du cinéaste avec le producteur de courts métrages Olivier Talpain naît l'envie de faire un film en commun. Tandis que Talpain, néophyte dans le domaine du long métrage de fiction, se charge de monter le financement, Edelstein commence à écrire. D’abord seul, puis avec l'aide notamment du scénariste et auteur de romans policiers Louis Sanders. Dans un souci de perfection, de nombreuses versions du scénario se succèdent et, selon Edelstein lui-même ${ }^{6}$, beaucoup de temps est perdu. Mais plus problématique à ses yeux reste le fait qu'ils travaillent dans un isolement absolu. En effet, le producteur, malgré son désir de bien faire, ignore tout des réseaux de production: "Dans un monde aussi clos, aussi petit que le cinéma, il faut tisser des réseaux de complicité, c'est extrêmement important. Il faut connaître les bonnes personnes.» Ce n'est qu'ainsi que la production peut donner au projet une plus-value qui sera déterminante: «Un projet défendu par un producteur qui a le respect de ses pairs est un projet qui bénéficie déjà d'une forme de considération.» Malgré tout, Edelstein ne regrette pas son choix. Il estime en revanche avoir sous-estimé les difficultés :

"Je ne me suis pas rendu compte que c'était un projet qui allait susciter de façon assez forte, je dirais, presque une forme de rejet qui tenait à la fois au fait que je n'avais plus fait de cinéma depuis vingt ans, et à la position marginale de mon producteur.»

En témoigne le fait qu'Edelstein, qui a travaillé à la télévision pendant plus de trente ans, n'obtient - dans un premier temps - aucune aide financière pour Quelques jours avant la nuit... La TSR refusera en outre de mettre à la disposition du cinéaste une des nombreuses caméras non utilisées durant le creux de l'été, l'obligeant à débourser CHF 10000.pour en louer une. Quelques jours avant la nuit... est donc un projet qui, dès le départ, n'a été soutenu d'aucune manière que ce soit. Budgété à environ un million et demi de francs suisses, il correspond à un "petit»
6 L'historique de production du film se base en très grande partie sur un entretien effectué le 24 juillet 2009 à Genève avec le cinéaste. Toutes les citations suivantes en sont tirées. 
Jean-Pierre Gos et Simon Edelstein sur le plateau de tournage

7 La subvention de la Ville de Genève permettra ensuite de toucher un pourcentage du Fonds Regio; la Loterie romande donnera également un subside.

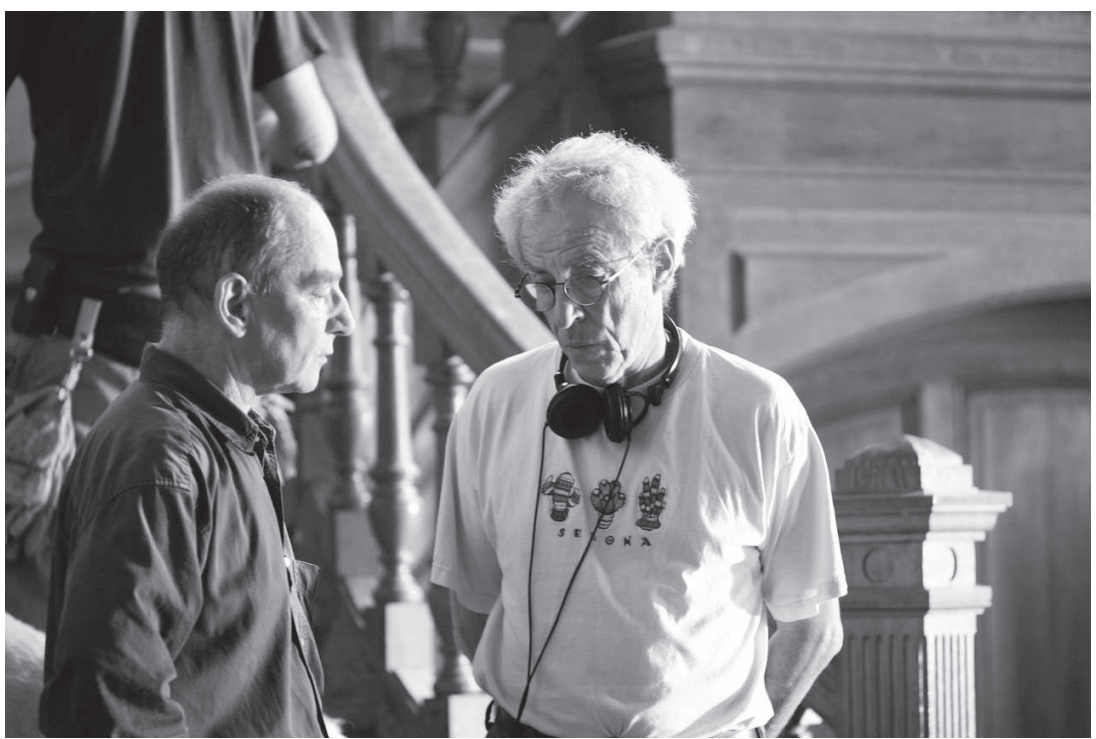

film selon les normes actuelles. Aucune aide au scénario ou à la production n’a été accordée de la part de Berne, ni de la télévision.

Edelstein, plutôt que de renoncer à son projet, estime qu'il vaut mieux faire le film et en chercher le financement après coup. Mais il a alors l'idée de le tourner sous forme de photographies, à la façon d'un roman-photo pour en faire baisser le coût. Or c'est la maquette de ce nouveau projet qui va relancer le processus de production. En effet, un nouveau scénario est soumis à la Ville de Genève qui se montre intéressée mais qui ne veut coopérer que si la forme du "roman-photo " est abandonnée. Le tir est rectifié et, chose inattendue, la Ville de Genève leur accorde le montant maximal de CHF 150000 .-, sans condition?. En tant que partenaire financier, il eût été en effet beaucoup plus normal que la Ville n'intervienne qu'à la fin du montage financier, pour venir compléter un budget déjà bouclé.

C'est avec cette somme que le tournage va se faire, ce qui implique de tourner très vite, de tout négocier au rabais (salaires, décors, etc.) et surtout de travailler (en vidéo) avec un nombre de collaborateurs minimal (absence notamment de directeur de production, de régisseur, et de perchman). En dehors d'Aldo Mugnier, chef opérateur expérimenté, d'un ingénieur du son et de deux machinistes, Edelstein est assisté de stagiaires de la Haute école d'art et de design (HEAD) de Genève qui n'ont jamais vu de plateau de tournage. Les acteurs, tous suisses, travaillent en semibénévolat et peuvent être appelés ailleurs à tout moment, ce qui veut 
dire que le cinéaste ne peut pas suivre véritablement de plan de tournage précis. Edelstein travaille au jour le jour en pensant constamment à une phrase de François Truffaut qui disait: "Avant de tourner un film, on rêve de faire un chef-d'œuvre, et quand on tourne, on n'a plus qu'une envie, c'est de le terminer.» En l'occurrence, le tournage est achevé en vingt-deux jours; le matériel est ensuite monté. Puis tout s'arrête faute d'argent. Or bien des choses restent encore à faire : bruitage, ambiance, musique, mixage et kinescopage du film en $35 \mathrm{~mm}$, ce qui représente environ CHF 250000.-. Approché, Philippe Berthet, chef de l'Unité Fiction de la TSR, promet de l'argent, puis se rétracte. Or le film a été proposé, non mixé, au festival de Soleure 2008 et retenu par Yvo Kummer, son directeur. Bloqué, Edelstein essaie de prendre contact avec un studio de mixage pour voir dans quelle mesure il est possible d'échelonner le paiement sur plusieurs années. Les deux parties jugent l'idée trop risquée et renoncent. Au mois de décembre 2007, Edelstein rencontre à nouveau Philippe Berthet et, arguant du fait qu'il est toujours très gênant de devoir dire aux responsables de Soleure que le film est bloqué car la TSR n'y a pas mis un centime (!), il parvient à convaincre Berthet de prendre en charge la musique et le mixage sous la forme d'un préachat du film ${ }^{8}$, complété par une petite aide substantielle. Le transfert sur pellicule ne sera en revanche pas effectué, le film n'existant à ce jour qu'en DVD.

\section{Sortie et réception}

Mais Quelques jours avant la nuit... peut finalement participer, sous cette forme, au festival de Soleure en janvier 2008: "Quand le film est sorti à Soleure, j'avais le sentiment que l'accueil avait été bon.» C'est ce que Freddy Buache confirmera d'ailleurs quelques mois plus tard, dans son propre compte rendu ${ }^{9}$. En l'occurrence, le journaliste de radio Patrick Ferla, enthousiasmé par le film, invite Edelstein à venir en parler le 25 janvier dans son émission «Presque rien sur presque tout»10. Puis, à l'exception d'un seul article paru dans L'Hebdo, toutes les critiques publiées à l'occasion de Soleure sont élogieuses:

"Réalisé avec peu de moyens financiers, [le dernier film de Simon Edelstein] conjugue un grand talent d'imagier, dont les cadrages et les mouvements de caméra tiennent de l'anthologie, et celui de quelques comédiens au mieux de leur inspiration, à commencer par Caroline Gasser et Jean-Pierre Gos, tous deux admirables. Dans l'esprit du cher Hitchcock, ce «suspense〉 psychologique dégage une inquiétante étrangeté qui tourne finalement à l'humour. Pas trace en tout cas de l'‘ennui> qu'on prête au cinéma suisse !»11
8 La TSR acquiert ainsi le droit de diffuser six fois le film sur tout le territoire suisse (deux diffusions par région linguistique).

9 Freddy Buache, "Le retour à la fiction de Simon Edelstein", Le Matin Dimanche, 8.11.2008: "Quelques jours avant la nuit gagna de nouveau le plus vif succès aux Journées de Soleure 2008 et trouve enfin sa présentation publique.".

10 "Un film complètement abouti, complètement réussi. [...]. Nous avons beaucoup, beaucoup, beaucoup aimé ce film!", Patrick Ferla, "Presque rien sur presque tout", Radio Suisse RSR 1, 25.01.2008.

11 Jean-Louis Kuffer, "Bonnes surprises à Soleure", 24 heures, 25.1.2008. 


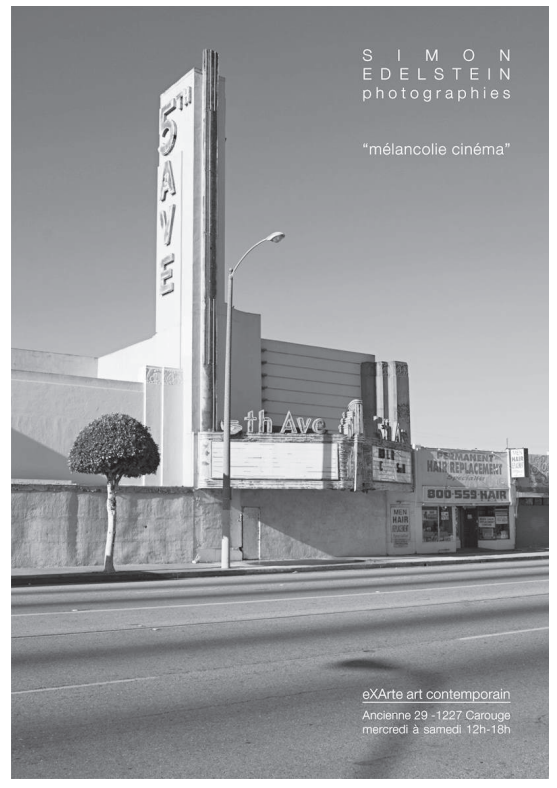

Carton d'invitation de l'exposition de photographies de Simon Edelstein, "Mélancolie cinéma "

12 Christoph Egger, "Zu Land und zu Wasser", NZZ, 28.1.2009: "Dass schwarzer Humor jedoch auch dezent und trotzdem wirkungsvollboshaft sein kann, wenn ein Meister Regie führt, demonstrierte Simon Edelsteins Quelques jours avant la nuit. Der Genfer, dessen erster Langspielfilm, Les vilaines manières, 1974 an den Solothurner Filmtagen warm aufgenommen worden war, bereitet sein Stück über eine nach einem Unfall offensichtlich ziemlichverwirrte 'berühmte Pianistin, als gekonnte Sunset Boulevard-Paraphrase mit einem Schuss Dinner for One auf, ohne je epigonal zu wirken." (notre traduction).

13 Antoine Duplan, L'Hebdo, 31.1.2008.

14 L'exposition "Mélancolie cinéma" présente ses photographies d'anciennes salles de cinéma prises à travers le monde (galerie eXArte art contemporain, 1227 Carouge, du 30 octobre au 29 novembre 2008).

15 Norbert Creutz, op. cit.

16 Antoine Duplan, op. cit.
Le critique de cinéma de la Neue Zürcher Zeitung livre de son côté un bilan du festival dans lequel le film d'Edelstein occupe une place tout à fait honorable:

"Le film de Simon Edelstein Quelques jours avant la nuit a prouvé que l'humour noir peut très bien être discret tout en étant méchamment efficace lorsqu'un maître en est le réalisateur. Le cinéaste genevois, dont le premier long métrage de fiction, Les Vilaines manières, avait trouvé un accueil chaleureux à Soleure en 1974, raconte l'histoire d'une ‘célèbre pianiste`, manifestement dérangée après un accident, en paraphrasant habilement Sunset Boulevard tout en y ajoutant un doigt de Dinner for One, sans toutefois jamais les imiter. »12

Et de fait, la presse alémanique dans son ensemble est positive. Une seule critique romande, nous l'avons dit, «descend» le film. Alors que le festival de Soleure vient de se terminer et que le film n'a pas encore été distribué, Antoine Duplan publie dans L'Hebdo le compte rendu suivant:

"Collaborateur de la TSR et cinéaste discret, Simon Edelstein a connu son heure de gloire en 1986, avec une adaptation un peu bancale mais pas honteuse de L'ogre, de Jacques Chessex, avant de connaître une longue éclipse. Avant la projection soleuroise, en guise de préambule maladroit, de récrimination déplacée ou de mise en garde un peu pathétique, le réalisateur lance: ‘Ce film s'est fait sans argent.> Cela se voit à l'écran. Mais ce huis clos au titre chabrolien et aux ambitions hitchcockiennes a aussi manqué d'inspiration et de savoir-faire. Rescapée d'un grave accident (bandage autour de la cheville), une pianiste cultive dans sa grande maison l'amour d'un fils absent. En fin de compte, seule la ténacité du cinéaste, qui a porté à terme ce projet branlant, force l'admiration.»13

Il est intéressant de relever ici - comme chez Creutz - la même volonté évidente de minimiser voire de passer sous silence toute la carrière d'Edelstein à la TSR en tant que réalisateur - sans parler de son œuvre de photographe $\mathbf{1 4}$-, et d'en faire un cinéaste «has been" en convoquant tout un lexique lié à la vieillesse ("cinéma de papa", "poussiéreux»15, «bancal», "longue éclipse», «branlant»16). Fait symptomatique de la réception critique dominante, un réalisateur n'existe que par ses longs métrages de fiction, genre noble, et tout autre mode de production n'est même pas envisageable. Enfin, il est frappant de voir que tous les journalistes évoquent les grands "auteurs» hollywoodiens (Hitchcock surtout), les «classiques» étant leur seule référence. Mais chez Creutz et Duplan, ceux-ci fonctionnent comme des parangons à suivre et ont valeur d'étalon dans leur appréhension des films d'aujourd'hui, ces derniers ne pouvant dans ce cas que rarement trouver grâce à leurs 
yeux. Chez les autres journalistes en revanche, la comparaison n'est jamais synonyme d'imitation servile mais source d'inspiration personnelle. Buache est celui qui le formule le plus clairement:

"Or cette impression ne constitue pas un aveu de faiblesse mais, au contraire, la résolution confirmée du refus des facilités à la mode pour une inscription dans une forme traditionnelle reprise de façon personnelle. $\gg \mathbf{1 7}$

Après Soleure, Mont-Blanc Distribution, une petite maison de distribution genevoise déjà impliquée dans la production du film, prend en charge sa diffusion. Souhaitant bénéficier des très bons échos critiques de la presse suisse alémanique, Mont-Blanc essaie de sortir le film d'abord en Suisse allemande. Elle présente donc le film à Thys Brunner, un des plus gros exploitants outre Sarine qui, bien qu'il apprécie le film, ne le prend pas. Le marché alémanique demeurant inaccessible, «un vent de déprime» s'abat sur tous les acteurs. Distributeur et producteur baissent les bras. Edelstein lui-même prend donc contact avec Rui Nogueira, directeur du Centre d'Animation Cinématographique de Genève, qui, aimant le film, va le programmer à partir du 3 novembre 2008 dans sa salle18. C'est à l'occasion de cette sortie que Norbert Creutz rend compte du film dans Le Temps. Son article sera repris trois fois dans ce quotidien, avant, après et le jour même de la sortie du film, via notamment le magazine culturel Sortir. Le film sort ensuite au Zinéma de Lausanne deux jours plus tard. Le nombre d'entrées est infime et sa carrière s'arrête là.

\section{Le rôle de la critique}

Interrogé sur le rôle qu’a joué la critique dans la carrière de son film, Simon Edelstein, lucide, ne veut pas surévaluer l'influence de cette dernière, mais estime qu'elle doit se faire dans certaines limites:

«Une bonne critique ne fait pas forcément venir des spectateurs. [...] Mais à partir du moment où, dans un pays tel que la Suisse, chaque film est un véritable combat, [...] il ne faut pas être humiliant, c'est-à-dire bénéficier d'une position dominante qui vous permet sans partage de dire ce que vous voulez. [...] C'est un travail, un film. Qu'on ne l'aime pas et qu'on le dise, c'est légitime [...], mais un film ne mérite jamais d'être méprisé.»

Mais pour Edelstein, la dérive critique la plus grave reste encore celle qu’a vécue son actrice principale Caroline Gasser. Contactée par le journaliste Michel Zendali qui lui dit avoir vu et aimé le film, elle accepte de venir en parler en direct à son émission de télévision Tard pour bar 19 . Zendali, qui remarque à plusieurs reprises que Quelques jours avant la
17 Freddy Buache, op. cit.

18 Le film est aussi programmé dans le cadre du festival Tout Ecran de Genève dès le 30 octobre 2008.

19 Emission du 6 novembre 2008, TSR 1, "James Bond partout, pourquoi ça marche encore?". 
20 Freddy Buache a signé une monographie du cinéaste en 2001 aux éditions L'Age d'homme.

21 Freddy Buache, op. cit.

\section{2 lbid.}

23 Norbert Creutz, op. cit.

24 Antoine Duplan, op. cit. nuit... n'a "vraiment rien à voir avec un James Bond", commence par demander à la comédienne de présenter brièvement le film avant de lui citer quelques extraits de la critique de Creutz, en guise de commentaires. Désarçonnée, la comédienne rétorque que la critique est "méchante» et se retrouve dans la situation désagréable de devoir défendre le film. Mais Zendali, à qui elle demandera pourquoi les «bonnes" critiques ne sont pas citées, ne dira jamais à l’antenne avoir aimé le film. Pour Edelstein qui n'a jamais été convié à l'émission de Zendali, c'est une véritable curée.

Précisant qu'il n'a jamais rencontré Duplan et Creutz, Edelstein pointe une fois encore l'importance des réseaux personnels entre cinéastes et journalistes pour légitimer et faire exister une œuvre. Meilleure preuve s'il en est, Buache est la seule personne qu'Edelstein connaisse personnellement. Ami et grand admirateur de Michel Soutter ${ }^{20}$, l'ancien directeur de la Cinémathèque suisse commence donc dans son article par prendre la peine de présenter Edelstein (en tant que photographe, puis opérateur attitré de Michel Soutter, enfin cinéaste), contrairement à la stratégie déployée par Creutz et Duplan. Puis, malgré le lien qui l'unit au cinéaste, Buache émet des critiques face à son film - «un scénario conventionnel à première vue trop exactement dosé » $\mathbf{2 1}$ notamment. Mais il nous livre, et c'est là son rôle, une analyse filmique proprement dite :

«Des notions d'insolite s'y développent donc moins au gré d'aventures de type romanesque ou de parcours dramatisé des sentiments pour se révéler par le décor, la position des personnages au centre du cadre et la présence très significative des objets. Un collier oublié sur les marches de l'escalier d'entrée, des bouquets de fleurs (éléments qui reviennent en refrain), les étoffes et vêtements désordonnés tirés d'une armoire suffisent à l'expression d'une situation où les portes se ferment avant de s'entrouvrir lentement, où les dialogues derrière le mur indiquent un appel de surprise derrière des apparences dites banales.» 22

Dire de la «mise en scène» qu'elle est «superficielle» ou du "point de vue» qu'il est "flottant» $\mathbf{2 3}$ ne décrit en revanche rien de concret, tout en servant au lecteur des formules lapidaires aux termes galvaudés et imprécis. Ou encore dire d'un cinéaste qu'il est "pathétique» ou "maladroit» $\mathbf{2 4}$ en tant que personne n'a pas sa place dans le cadre d'une critique cinématographique.

Enfin, et c'est là peut-être le plus important, il faudrait toujours garder à l'esprit que les films suisses sont de petits objets artisanaux, fragiles, 
et que, lorsqu'ils sortent sur territoire national, la critique locale a un impact plus important que lorsqu'elle rend compte d'un blockbuster américain. Face à un film suisse, le grand public n'a très souvent plus ses repères habituels (grands noms d'acteurs, de cinéastes) pour se décider. Le rôle de la critique est alors à double tranchant: soit elle déclenche un bouche-à-oreille qui éveille la curiosité des spectateurs, soit elle les décourage d'emblée de se déplacer. On ne peut donc que s'étonner de l'acharnement dont certains journalistes ont fait preuve à l'égard d'un film qui n'a eu, somme toute, qu'une sortie très marginale.

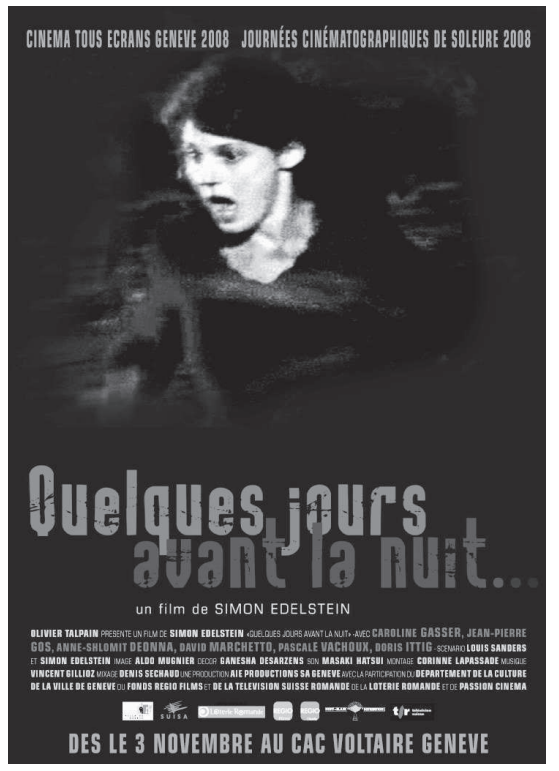

Quelques jours avant la nuit... (2008, 94 min.)

Réalisation: Simon Edelstein. Production: Olivier Talpain, Aïe Production SA, Genève. Image: Aldo Mugnier. Scénaristes: Louis Sanders et Simon Edelstein. Son: Masaki Hatsui. Montage: Corinne Lapassade. Musique: Vincent Gilloz. Interprétation: Caroline Gasser (Anne), JeanPierre Gos (Gilbert), Anne-Shlomit Deonna (Vilma), David Marchetto (Frank), Pascale Vachoux (Catherine), Doris Ittig (mère de Marguerite). 